\title{
Action des taons (Diptera, Tabanidae) sur le comportement d'un troupeau de zébus au pâturage en Guyane française
}

\author{
H.L. RAYMOND \\ INRA, Unité de Zoologie, \\ Direction des Services vétérinaires, \\ F 97306 Cayenne Cedex, Guyane française
}

\begin{abstract}
Résumé
Les taons les plus nuisibles dans les savanes de Guyane française sont Cryptotylus unicolor (Wiedemann) au milieu de la saison sèche et Tabanus importunus Wiedemann en fin de saison sèche. La première espèce n'est active que pendant une $1 / 2$ heure à l'aube et au crépuscule mais elle dérange considérablement le bétail. Quand le nombre de taons croît, en fin d'après-midi, les animaux se regroupent, cessent de pâturer et se couchent. La seconde espèce pique toute la journée mais est plus abondante en fin d'après-midi et empêche le pâturage plusieurs heures par jour. Le rythme des battements de queue augmente avec le nombre de taons. L'utilisation de ce rythme comme indice d'abondance des insectes est discutée.
\end{abstract}

Mots clés : Bovins, Tabanidae, Guyane française, relation hôte-parasite, comportement alimentaire, comportement de confort.

\section{Introduction}

Les taons sont les ectoparasites les plus nuisibles aux bovins en Guyane française (Raymond et al., 1987). En Amérique du Nord, la présence de 4 taons par tête de bétail peut réduire de $18 \%$ le gain moyen quotidien pondéral de jeunes bovins à viande (Bruce \& DECKER, 1951). Une densité apparemment faible d'insectes piqueurs peut donc avoir une incidence économique. Les pertes de production dues aux insectes piqueurs peuvent résulter de la spoliation sanguine (Hollander \& WRIGHT, 1980) mais aussi des modifications du comportement des bovins (Harvey \& Launchbaugh, 1982). L'observation de ce comportement peut donc être très utile pour l'étude des relations entre taons et bovins. Un travail préliminaire (RAYMOND \& Rousseau, 1987) avait été réalisé en Guyane française sur les bovins de race créole élevés à la longe en zone suburbaine boisée. Les observations présentées ici, concernant un troupeau de zébus élevé sur prairies artificielles en région de savane, devaient répondre aux questions suivantes: 1) Quelles sont les espèces de taons les plus nuisibles aux bovins dans les savanes de Guyane ? 2) Comment réagissent les bovins aux attaques de ces taons ? 3) Peut-on évaluer l'abondance des taons à partir du comportement du bétail, ce qui permettrait peut-être une lutte plus efficace contre ces parasites ? 


\section{Conditions d'observation}

Le troupeau de zébus étudié, comprenant un taureau et une vingtaine de vaches gestantes, pâturait en rotation diverses parcelles de la ferme de Savane Combi, près de Sinnamary (à $100 \mathrm{~km}$ au nord-ouest de la ville de Cayenne). Les observations ont été effectuées pendant la saison sèche (de juillet à décembre 1983), période d'abondance maximale des taons, et au moment où ceux-ci sont les plus nombreux, c'est-à-dire en fin d'après-midi (RAYMOND et al., 1987).

Treize séances d'observation de 2 heures ont été réalisées (à raison de 1 à 4 séances par mois). Les séances débutaient à $17 \mathrm{~h}$ avant le 20 octobre et à $17 \mathrm{~h} 40$ après cette date. Ainsi, la fin de la séance coïncidait-elle approximativement avec la tombée de la nuit et la disparition subite des taons, qui succédait immédiatement à leur pic d'activité. Les paramètres concernant l'ensemble du troupeau sont notés toutes les $10 \mathrm{mn}$ : niveau d'agrégation (animaux groupés, épars ou très épars), proportion d'animaux pâturant ou ruminant, attitude (nombre d'animaux couchés ou debout). De plus, pendant chaque séquence de 10 minutes, 4 à 6 vaches sont observées successivement durant environ une minute afin de déterminer le nombre de taons posés et visibles sur chacune d'elles (cette estimation est donc relative) et la fréquence des battements de leur queue. Les pourcentages d'animaux se livrant aux diverses activités et les moyennes du nombre de taons et de la fréquence des battements de queue sont calculés pour chaque séquence de $10 \mathrm{mn}$.

Des taons ont été capturés pendant chaque séance d'observation à l'aide d'un piège de Malaise (1937) comportant une tente en moustiquaire verte, un leurre visuel (panneaux noirs posés sous la tente simulant le corps d'un gros herbivore) et un appât olfactif (dégagement de gaz carbonique simulant la respiration de l'animal). Ce piège et ses performances sont décrits dans un autre article (RAYMOND, 1988). Le piège est généralement relevé en fin de séance, après $2 \mathrm{~h}$ de fonctionnement. Au cours de certaines séances, des relevés ont été effectués toutes les $30 \mathrm{mn}$ pour évaluer les variations horaires de l'abondance des taons, exprimée en insectes pris par piège et par heure de capture. Ce piège a été aussi utilisé sans appât au gaz carbonique pour des captures de $24 \mathrm{~h}$ (les insectes étant récoltés à la tombée de la nuit) mais il donne alors des échantillons moins représentatifs des populations de taons piquant les animaux. L'abondance relative des taons est alors exprimée en insectes pris par piège et par jour de capture. Les taons ont été identifiés à l'aide d'une collection de spécimens de Guyane française déterminés par le $P^{r}$ G.B. FaIRChILD (Université de Floride, Gainesville). L'interprétation statistique a été effectuée selon les méthodes classiques (SchWARTZ, 1963).

\section{Résultats}

\section{A. Effet de la date}

En juillet, les taons sont peu nombreux dans les pièges (26 taons par heure avec $\mathrm{CO}_{2}$ et 9 par jour sans $\mathrm{CO}_{2}$ ) et sur les animaux ; la fréquence des battements de queue 


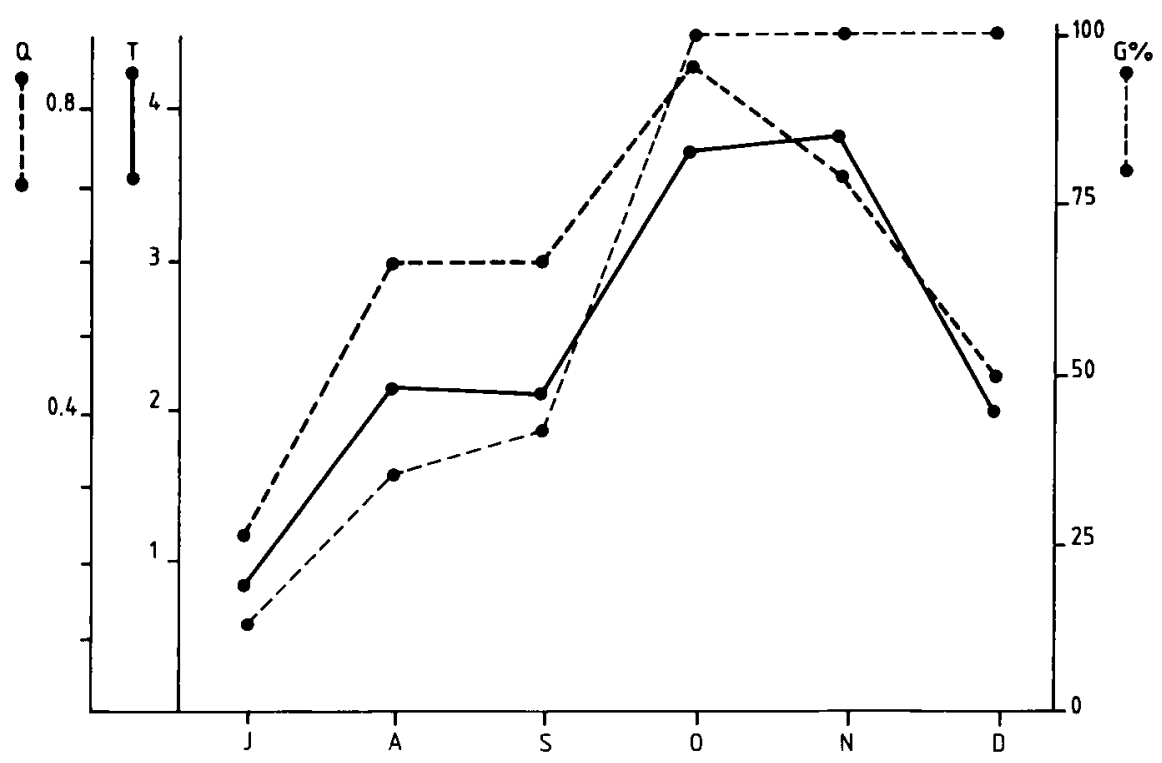

FIG. 1

Moyennes mensuelles (juillet-décembre) du nombre de taons observés par animal ( $T$ ), de la fréquence des battements de queue des animaux $(Q$, coups par seconde) et pourcentage d'observations pendant lesquelles les animaux sont groupés $(G)$.

Monthly means (July-December) of the number of horse-flies observed per animal $(T)$, of the rhythm of tail switching ( $Q$, strokes per second) and percentage of observations during which the herd aggregated $(G)$.

est faible (fig. 1). Les animaux, rarement groupés ou couchés, sont en majorité en train de pâturer pendant la période d'observation (fig. 2). D'août à octobre, le nombre de taons (200 à 300 taons $/ \mathrm{h}$ et 30 à 40 taons $/ \mathrm{j}$ suivant le piège) et la fréquence des battements de queue croissent. Les animaux, plus souvent couchés ou groupés, pâturent moins. D'octobre à décembre, la plupart des paramètres mesurant l'abondance des taons (nombre d'insectes observés sur l'animal en fin d'après-midi, pris au piège Malaise appâté au gaz carbonique en fin d'après-midi ou pris au piège Malaise sans appât au gaz carbonique pendant la journée) et le dérangement des animaux (fréquence des battements de queue, pourcentage d'observations pendant lesquelles les animaux sont groupés, pourcentage d'animaux couchés) prennent leurs valeurs maximales. En novembre les captures de taons dépassent respectivement 300 taons $/ \mathrm{h}$ et $100 \mathrm{taons} / \mathrm{j}$. Par contre, le pourcentage d'animaux ruminant au moment des observations est plus élevé d'octobre à décembre que de juillet à septembre. La période d'observation peut être divisée en deux phases : de juillet à septembre l'abondance des taons et l'intensité des réactions des animaux (fig. 1), dont la majorité pâturent (fig. 2), sont en moyenne modérées ; d'octobre à décembre, l'abondance des taons et l'intensité des réactions des animaux, qui pâturent peu, sont très fortes. 


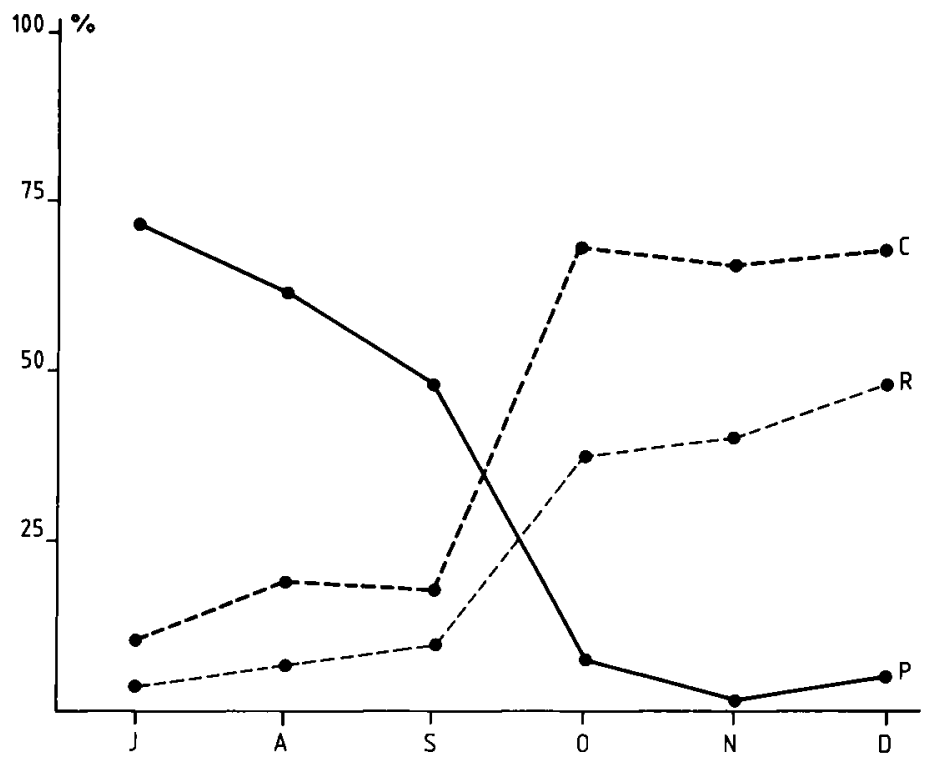

FiG. 2

Pourcentages mensuels (juillet-décembre) d'animaux pâturant $(P)$, ruminant $(R)$ et couchés $(C)$. Monthly percentages (July-December) of grazing $(P)$, ruminating $(R)$ and lying $(C)$ animals.

Tableau 1

Analyse statistique (analyse de variance) des effets de l'heure et du mois sur les variables étudiées (nombre de taons par animal: $T$, fréquence des battements de queue: $Q$, pourcentage d'observations pendant lesquelles les animaux sont groupés: $G$,

pourcentages d'animaux pâturant $: P$, ruminant $: R$, couchés : $C$, seuils de signification : nombres : non significatif : N.S.) pour le premier (juillet, août, septembre) et le second (octobre, novembre, décembre) trimestres de la saison sèche.

Statistical analysis (analysis of variance) of the influences of time and month on the studied variable $(T:$ number of horseflies per animal $Q$ : tail switching rhythm; $G$ : percentage of observations during which the herd aggregated, percentages of grazing animals : $P$, ruminating $: R$, lying : $C$; significance levels : numbers; not significant: N.S.) for the first (July, August, September) and the second (October, November, December) quarters of the dry season.

\begin{tabular}{|c|c|c|c|c|}
\hline Facteurs & Heure & & Mois & \\
\hline \multirow[t]{3}{*}{ Trimestre } & Juillet & Octobre & Juillet & Octobre \\
\hline & Août & Novembre & Août & Novembre \\
\hline & Septembre & Décembre & Septembre & Décembre \\
\hline \multicolumn{5}{|l|}{ Variables } \\
\hline $\mathrm{T} \ldots \ldots$ & 0,001 & N.S. & 0,025 & 0,05 \\
\hline $\mathrm{Q} \ldots \ldots \ldots$ & 0,001 & 0,001 & 0,01 & 0,001 \\
\hline $\mathrm{G} \ldots \ldots$ & 0,001 & - & 0,05 & - \\
\hline $\mathrm{P} \ldots \ldots \ldots$ & 0,001 & N.S. & 0,05 & N.S. \\
\hline $\mathrm{R} \ldots \ldots \ldots$ & 0,001 & 0,05 & N.S. & 0,01 \\
\hline$C \ldots \ldots$ & 0,001 & 0,05 & N.S. & N.S. \\
\hline
\end{tabular}




\section{B. Effet de l'heure}

L'effet de l'heure, très hautement significatif pour toutes les variables étudiées de juillet à septembre, est moins marqué d'octobre à décembre (tabl. 1). Au cours du premier trimestre, le nombre de taons par animal et la fréquence des battements de queue augmentent parallèlement au cours de l'après-midi ; les animaux se regroupent (fig. 3), se couchent et cessent de pâturer mais peu d'entre eux ruminent (fig. 4). Cryptotylus unicolor (Wiedemann) est le taon le plus abondant au crépuscule. Cette espèce de grande taille (17 mm environ), piquant le dos et les flancs, irrite beaucoup les animaux (sa piqûre est très douloureuse pour l'homme). Les mouvements de défense du bétail chassent souvent cette espèce, interrompant son repas de sang mais elle repique aussitôt une autre partie du corps ou un autre animal.

D'octobre à décembre, le nombre de taons par animal et la fréquence des battements de queue sont élevés dès le début des séances d'observation, à $16 \mathrm{~h} 40$ ou $17 \mathrm{~h}$, et ces variables augmentent fortement une demi-heure avant la tombée de la nuit ; les animaux, groupés pendant toute la durée des observations (fig. 5), pâturent très peu et le pourcentage de ceux qui ruminent diminue en cours d'après-midi ; la majorité des animaux sont couchés; ils se relèvent au crépuscule mais ne recommencent pas à pâturer immédiatemment (fig. 6). Des observations qualitatives faites à la tombée de la nuit (quand l'obscurité interrompt les observations quantitatives) indiquent que les bovins recommencent brusquement à pâturer dès que l'activité des taons a cessé. Les pièges appâtés au gaz carbonique, relevés de demi-heure en demi-heure, indiquent aussi une croissance de l'abondance des taons en fin d'après-midi. Pour la période d'octobre à décembre, le nombre moyen de Tabanus importunus Wiedemann (l'espèce la plus abondante et la plus grosse, environ $20 \mathrm{~mm}$ de long) capturés par un piège est égal à 31,3 entre $16 \mathrm{~h} 40$ et $17 \mathrm{~h} 10$, à 49,7 entre $17 \mathrm{~h} 10$ et $17 \mathrm{~h} 40$, à 70,3 entre $17 \mathrm{~h} 40$ et $18 \mathrm{~h} 10$ et à 81,3 entre $18 \mathrm{~h} 10$ et $18 \mathrm{~h} 40$. Cette espèce est active pendant la journée, contrairement à Cryptotylus unicolor.

\section{Abondance des taons}

Les moyennes mensuelles du nombre de taons par animal, du nombre de taons pris par heure de capture au piège à leurre visuel et à appât olfactif et du nombre de taons pris par jour de capture réalisée avec un piège à leurre visuel mais dépourvu d'appât olfactif (moins efficace que le piège muni d'un appât olfactif) ont été comparées. Une corrélation linéaire a été mise en évidence entre le nombre $T$ de taons observés sur l'animal et le nombre $T_{P}$ de taons pris par heure de capture au piège à leurre visuel et à appât olfactif $\left(\mathrm{r}=0,88 ; 4\right.$ d.d.1. ; risque inf. à 0,$\left.05 ; \mathrm{T}_{\mathbf{P}}=74,6 \mathrm{~T}-0,5\right)$. Aucune liaison significative ne peut être établie dans le cas du piège dépourvu d'appât olfactif. Les résultats des piègeages confirment la prédominance de Cryptotylus unicolor en début de saison sèche (66\% des taons récoltés) et celle de Tabanus importunus en fin de saison sèche (54\% des taons récoltés).

\section{Réactions du bétail}

Une corrélation linéaire entre le nombre $\mathrm{T}$ de taons par animal et la fréquence $\mathrm{Q}$ des battements de queue a été établie sur la totalité des séquences d'observation de $10 \mathrm{mn}(\mathrm{r}=0,84 ; 144$ d.d.1. ; risque inf. à 0,$001 ; \mathrm{Q}=0,16 \mathrm{~T}+0,19)$. L'estimation de 


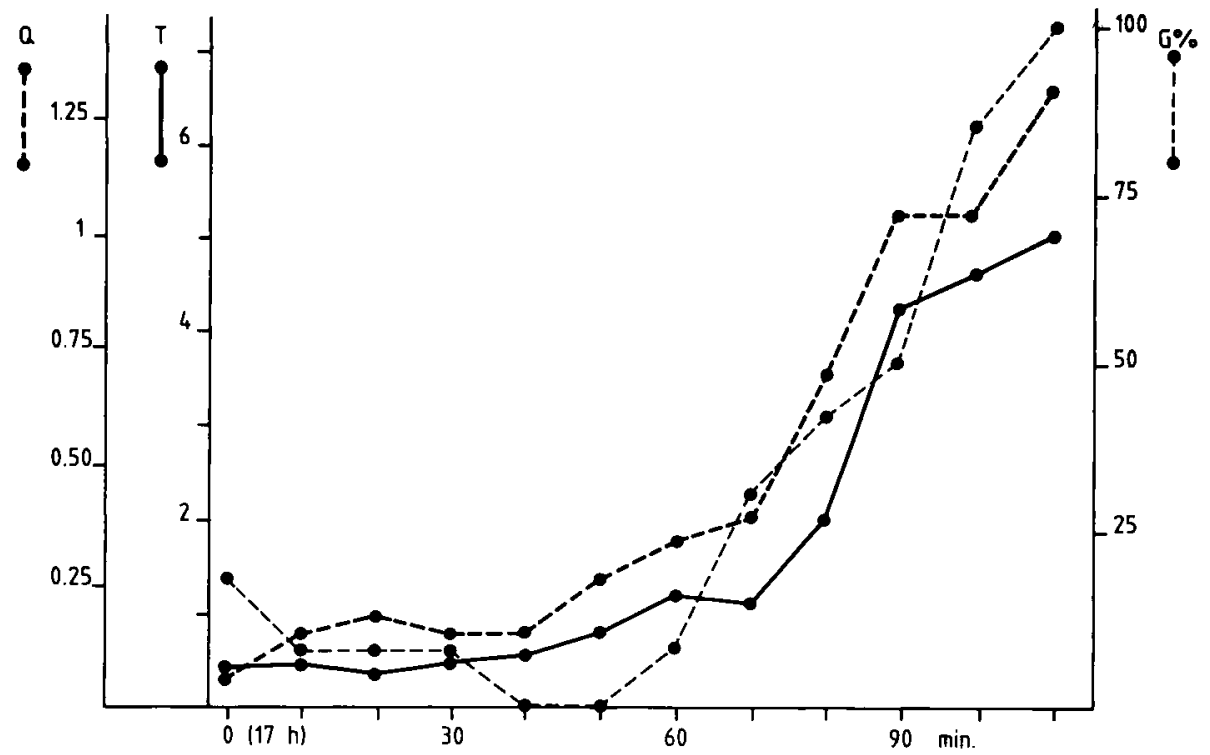

Fig. 3

Nombre de taons par animal $(T)$, fréquence des battements de queue $(Q)$ et pourcentage d'observations pendant lesquelles les animaux sont groupés (G) au cours des 120 minutes précédant la nuit (moyennes pour 12 séquences de $10 \mathrm{mn}$; mois de juillet, août et septembre).

Number of horseflies observed per animal $(T)$, rhythm of tail switching $(Q)$ and percentage of observations during which the herd aggregated $(G)$, during the $120 \mathrm{~min}$ preceding the night (means for 12 sequences of $10 \mathrm{~min}$;

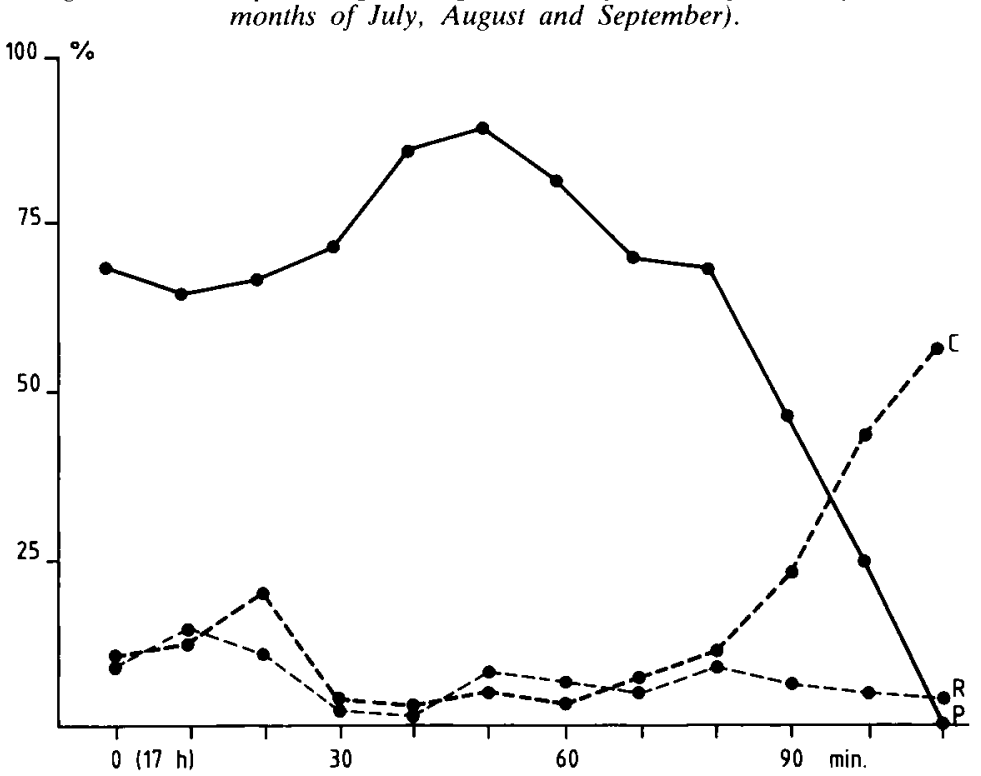

Fig. 4

Pourcentages d'animaux pâturant $(P)$, ruminant $(R)$ et couchés $(C)$ au cours des 120 mn précédant la nuit (juillet-septembre).

Percentages of grazing $(P)$, ruminating $(R)$ and lying $(C)$ animals during the 120 min preceding the night (July-September). 


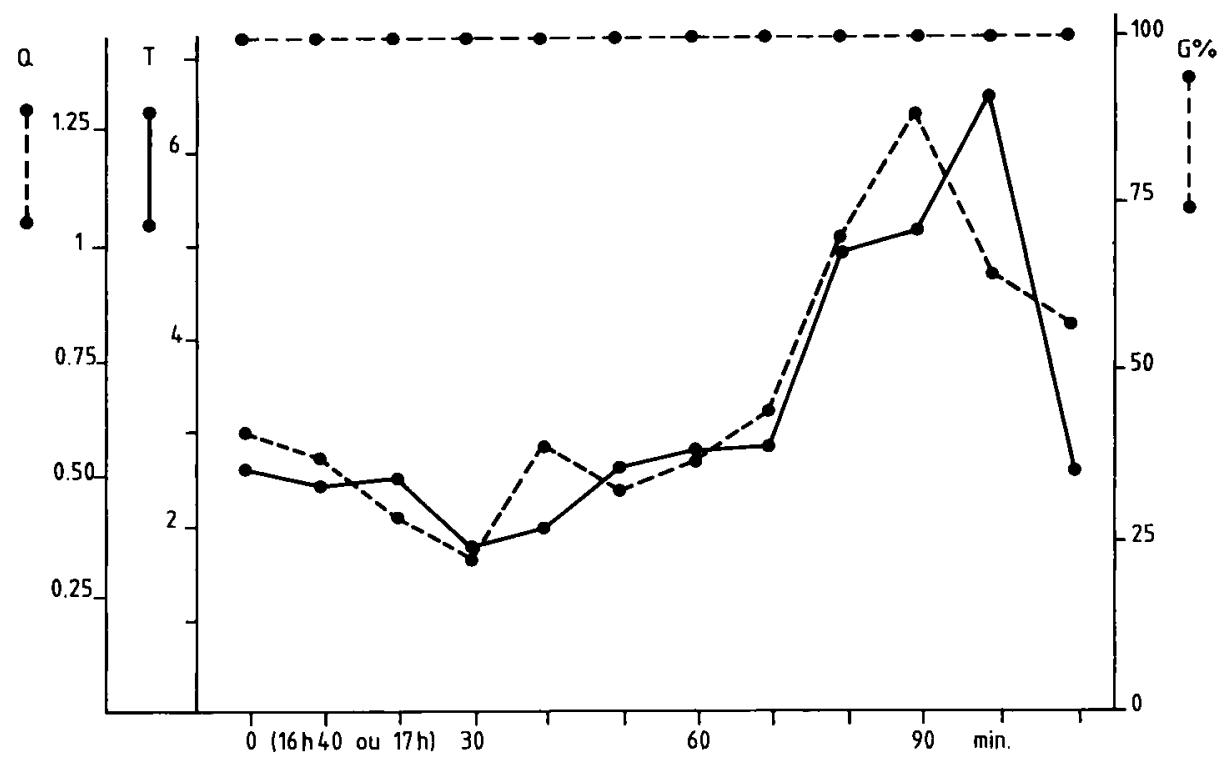

FIG. 5

Nombre de taons par animal ( $T)$, fréquence des battements de queue $(Q)$

et pourcentage d'observations pendant lesquelles les animaux sont groupés $(G)$ au cours des $120 \mathrm{mn}$ précédant la nuit (moyennes de 12 séquences de $10 \mathrm{mn}$; mois d'octobre, novembre et décembre; observations débutant à $16 \mathrm{~h} 40$ ou $17 \mathrm{~h}$ suivant l'heure de coucher du soleil).

Number of horseflies per animal (T), rhythm of tail switching $(Q)$

and percentage of observations during which the herd is aggregated $(G)$, during the $120 \mathrm{~min}$ preceding the night (means for 12 sequences of 10 min; months of October, November and December; observations beginning at 16.40 or 17.00 hours, according to sunset (time).

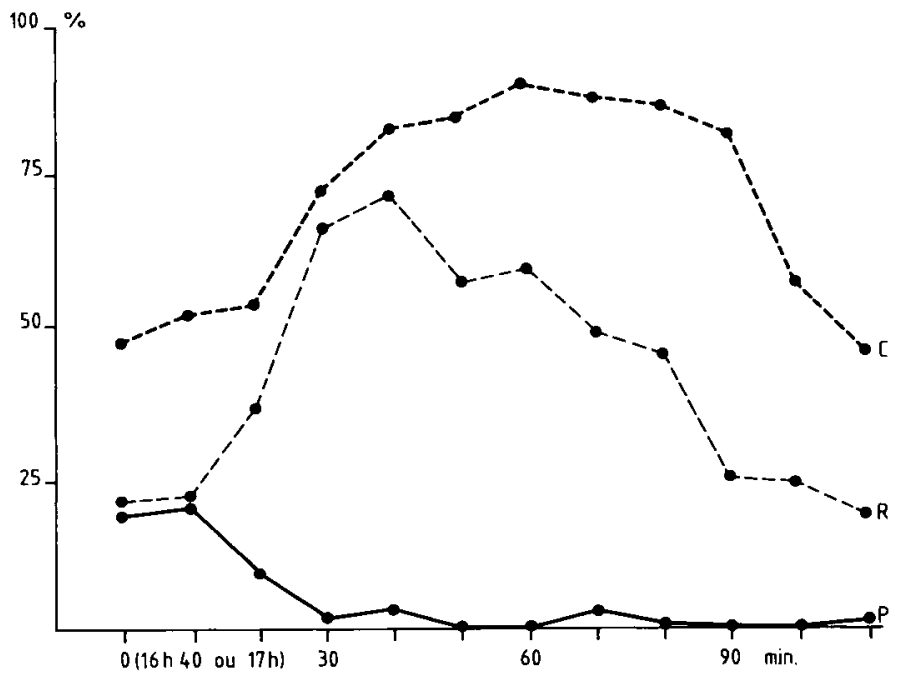

Fig, 6

Pourcentages d'animaux pâturant $(P)$, ruminant $(R)$ et couchés $(C)$ au cours des $120 \mathrm{mn}$ précédant la nuit (octobre-décembre).

Percentages of grazing $(P)$, ruminating $(R)$ and lying $(C)$ animals during the $120 \mathrm{mn}$ preceding the night (October-December). 


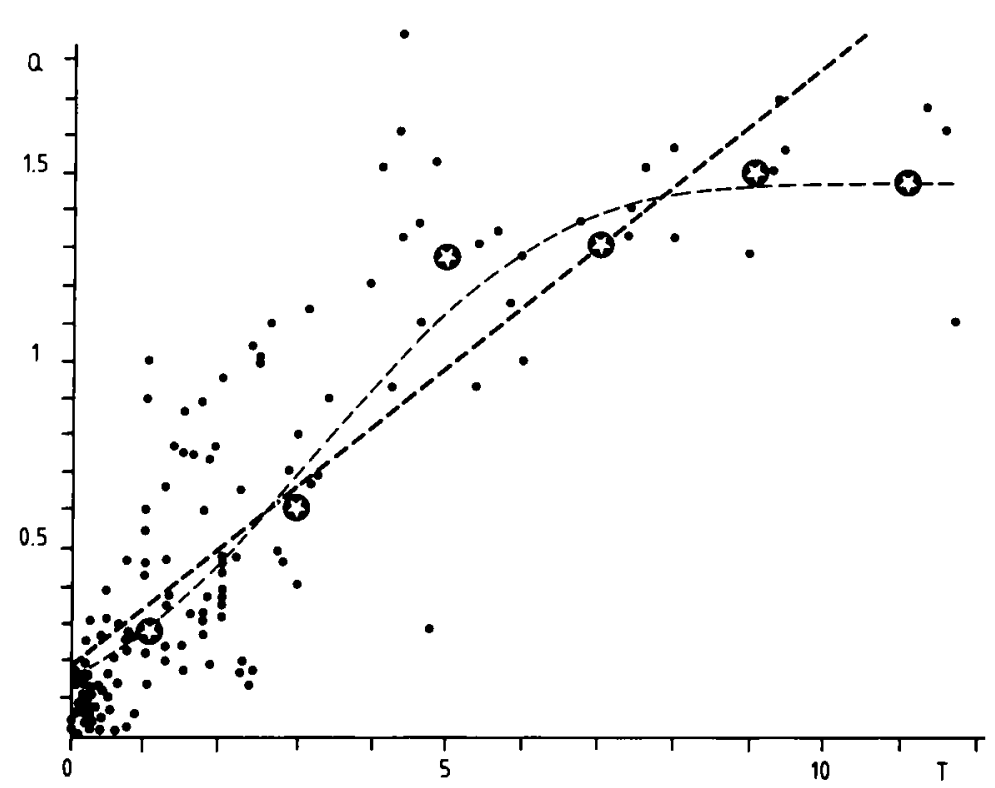

Fig. 7

Relation entre le nombre de taons par animal $(T)$ et la fréquence des battements de queue $(Q)$ pour la totalité des séquences (points), droite de régression (tireté gras; $r=0,84 ; 144$ d.d.l., risque inf. à 0,001), moyennes de $Q$ pour 6 classes de $T$ (étoiles), ajustement des moyennes de $Q$ aux médianes des classes de T par une courbe logistique (tireté fin ; $r=0,95 ; 4$ d.d.l. ; risque inf. à 0,01 ).

Relationship between the number of horseflies per animal $(T)$ and rhythm of tail switching $(Q)$ for all the sequences (points), regression (broken line, bold; $r=0.84 ; 144$ d.f. significant at the 0.001 level), means of $Q$ for 6 classes of $T$ (stars), fitting of the means of $Q$ to the medians of $T$ classes by a logistic curve (broken line, fine ; $r=0.95 ; 4$ d.f.; significant at the 0.01 level).

$Q$ par une fonction logistique ou exponentielle ne permet pas de meilleur ajustement aux données obtenues sur l'ensemble des séquences. Par contre si les valeurs de $\mathrm{T}$ sont réparties en 6 classes d'amplitude 2, d'effectifs inégaux et comprises entre 0 et 12 taons par animal et si les moyennes de $Q$ sont calculées pour chaque classe, un modèle logistique (fig. 7) permet un meilleur ajustement entre les médianes de $T$ et les moyennes de $\mathrm{Q}$. Une densité de 5 taons par animal correspond approximativement à une fréquence de 1 coup de queue par seconde, valeurs surtout observées en novembre (fig. 1). En début de saison sèche, de telles valeurs ne sont observées qu'au crépuscule (fig. 3). Le pourcentage d'animaux pâturant diminue quand la densité de taons dépasse 2 insectes par animal. La fréquence des battements de queue est alors supérieure à 0,5 .

\section{Discussion}

Chez les chevaux de Camargue, le temps de pâturage diminue et les mouvements de confort sont plus fréquents pendant la période d'activité des taons (Boy \& DUNCAN, 1979). Les chevaux se regroupent au moment des attaques de taons pour réduire les 
nuisances infligées par les insectes (DuCAN \& Vigne, 1979 ; Hughes et al., 1981). Chez les bovins, le maximum de la fréquence des battements de queue coïncide avec le pic d'activité de la mouche piqueuse Haematobia (Muscidae) et cette fréquence diminue quand les animaux sont traités à l'aide de sacs-poudreurs contenant du stirofos (HARVEY \& Launchbaugh, 1982). Les résultats obtenus en Guyane sont en accord avec ces observations. Rousseau (1982) a constaté que le traitement des troupeaux de bovins contre Tabanus importunus par aérosols thermiques de deltaméthrine réduisait la fréquence des battements de queue $(0,77$ battement par seconde avant traitement et 0,30 après).

Des bovins isolés à la longe sont beaucoup plus piqués que les zébus du troupeau étudié ici (jusqu'à 150 taons piquant simultanément un animal) et la fréquence des battements de queue de ces animaux varie considérablement en fonction de l'individu (RAymond \& Rousseau, 1987). Dans cette étude, le rassemblement du troupeau sous l'effet des insectes, l'appartenance des animaux à une même catégorie (femelles gestantes), l'homogénéité relative de la prairie où ils peuvent se déplacer librement et les interactions sociales (déplacements des animaux s'effectuant généralement en groupe ; compétition entre individus pour les places à l'intérieur du groupe, les mieux protégées contre les taons) uniformisent probablement le nombre de taons piquant les animaux et l'intensité de leurs réactions contre ces insectes.

Le fait que les animaux se couchent ou ruminent à certaines heures n'est évidemment pas uniquement lié à la présence des taons. En climat tropical humide, les bovins pâturent de préférence tôt le matin et en fin d'après-midi (Williamson \& Payne, 1978). Les zébus de Guyane française, quand les taons sont rares (en saison des pluies, en "saison sèche" exceptionnellement pluvieuse ou dans les élevages installés sur défrichement forestier) sont couchés et ruminent (éventuellement à l'ombre) en milieu de journée, pâturent dans la matinée et l'après-midi mais sont agités au crépuscule (BARBIER, 1988). Le rassemblement du troupeau est une réponse plus caractéristique se produisant quand les insectes deviennent plus nombreux. Ce type de réponse permet de savoir que l'abondance des taons dépasse un seuil critique (ici voisin de 2 insectes par animal). La fréquence des battements de queue est sans doute le meilleur indice de l'abondance des insectes car cette réaction est destinée à les chasser et son intensité peut varier dans une gamme étendue. Pour le troupeau et le milieu étudiés, cette fréquence est liée au nombre de taons par animal, quelles que soient l'heure et la date. Une abondance de 2,5 taons par animal, correspondant approximativement au rythme d'un coup de queue par période de deux secondes, pourrait être considérée comme un seuil de nuisance. Des observations prolongées dans d'autres milieux seraient nécessaires pour confirmer la validité d'un tel seuil.

\section{Conclusions}

Deux espèces de taons sont particulièrement nuisibles en saison sèche dans les savanes de Guyane française en raison de leur abondance, de leur taille et de leur agressivité. C. unicolor n'est actif qu'à l'aube et au crépuscule en août et en septembre. T. importunus est présent presque toute la journée d'octobre à décembre.

Les bovins réagissent aux attaques de taons par une intensification des mouvements de défense contre les insectes (battements de queue), un rassemblement du troupeau et une modification des rythmes d'activité alimentaire. 
Les captures crépusculaires au piège à leurre visuel et appât olfactif permettent une évaluation de l'activité des taons. La mesure de la fréquence des battements de queue des animaux au pâturage fournit aussi une estimation indirecte de l'abondance des taons.

Cet ensemble d'observations confirme certaines données obtenues pour d'autres herbivores (chevaux) ou d'autres insectes (Muscidae) dans d'autres régions (Europe et Amérique du Nord) et constitue la première étude quantitative des réactions d'un troupeau de bovins au pâturage aux attaques des taons (Tabanidae).

Reçu en avril 1987.

Accepté en décembre 1987.

\author{
Summary \\ Effect of horseflies (Tabanidae, Diptera) on the behaviour \\ of a grazing zebu herd in French Guiana
}

\begin{abstract}
The influence of the abundance of horse-flies (number of flies observed per cow) on some behavioural data (percentage of cows grazing, ruminating or lying, frequency of herd aggregation, rhythm of tail switching) was studied in a small zebu herd during the periods of highest activity of flies (dry season, July-December and late afternoon, 16.40-19.00 hours in a heavily infested area (artificial pastures surrounded by coastal savannah). The activity of flies, the rhythm of tail switching, the frequency of herd aggregation (fig. 1) and the percentages of lying and ruminating animals (fig. 2) were low in July and increased during the second part of the dry season. The percentage of grazing animals decreased between July and December. The values recorded, depended on the time in the first part of the dry season strongly (Table 1), during which the most noxious fly was Cryptotylus unicolor (Wied.). This fly bit only at dusk, caused a sharp increase in the tail switching rhythm, herd aggregation (fig. 3) and percentage of lying animals and reduces the number of grazing cows (fig. 4). Tabanus importunus Wied., the most abundant fly in late dry season, bit during the whole observation period, inducing permanently the aggregation of the herd (fig. 5) and inhibiting the grazing (fig. 6). The activity of this fly increased in late afternoon, disturbing more severely the animals. The tail switching rhythm increased with the number of flies observed on the cows (fig. 7) and its use as insect abundance index is discussed.
\end{abstract}

Key Words : Cattle, Tabanidae, French Guiana, host-parasite relationship, grazing behaviour, comfort behaviour.

\title{
Références bibliographiques
}

Barbier M.F., 1988. Comportement alimentaire des zébus au pâturage en Guyane. Rev. Elev. Méd. vét. Pays trop., (sous presse).

Boy V. \& Duncan P., 1979. Time-budgets of Camargue horses. I. Developmental changes in the time-budgets of foals. Behaviour, 71, 187-202.

Bruce W.N. \& Decker G.C., 1951. Tabanid control on dairy and beef cattle with synergized pyrethrins. J. econ. Entomol., 44, 154-159.

DUNCAN P. \& VIGNE N., 1979. The effect of group size in horses on the rate of attacks by bloodsucking flies. Anim. Behaviour, 27, 623-625. 
Harvey T.L. \& Launchbaugh J.L., 1982. Effect of horn flies on behavior of cattle. $J$. econ. Entomol., 75, 25-27.

Hollander A.L. \& Wright R.E., 1980. Impact of tabanids on cattle blood meal size and preferred feeding sites. J. econ. Etomol, 73, 413-433.

Hughes R.D., Duncan P. \& Dawson J., 1981. Interaction between Camargue horses and horseflies (Diptera, Tabanidae). Bull. ent. Res., 71, 227-242.

Malaise R., 1937. A new insect trap, Entomol, Tidskrift, 58, 148-160.

Raymond H.L., 1988. Intérêt des pièges de Malaise appâtés au gaz carbonique pour l'étude des taons crépusculaires (Diptera, Tabanidae) de Guyane française. Insect. Sci. Applic. (sous presse).

Raymond H.L. \& Rousseau F., 1987. Abondance des taons (Diptera, Tabanidae) et réactions des bovins dans un élevage traditionnel de Guyane française. Acta Oecologica Oecol. Applic., 8, $125-134$.

Raymond H.L., Barre N. \& Camus E., 1987. Données nouvelles sur les taons (Diptera, Tabanidae) et les tiques (Acari, Ixodidae) de Guyane française [in] Systèmes d'élevage herbager en milieu équatorial, Séminaire INRA-ORSTOM-CIRAD, Cayenne, 9-10 décembre 1985: 321-334, INRA Publ., Paris.

Rousseau F., 1982. Contribution à l'étude des Tabanidés de Guyane française: Protection du bétail par brouillard insecticide. Thèse doc. vét. Univ. Cl. Bernard, Lyon, 124 p.

Schwartz D., 1963. Méthodes statistiques à l'usage des médecins et des biologistes. Flammarion, Paris, $296 \mathrm{p}$.

Williamson G. \& Payne W.J.A., 1978. An introduction to animal husbandry in the tropics (3d ed.). Longman, London \& New York, $755 \mathrm{p}$. 